Prostate cancer is one of the most commonly diagnosed malignancies in males. Surgery and radiotherapy are primary methods of curative treatment in patients with this diagnosis. Development of technology and long-term clinical experience enable optimisation of radiotherapy for all clinical stages of the disease. The purpose of this paper is to present general principles of treatment selection and conduct in prostate cancer patients to doctors who are not specialists in medical oncology or radiotherapy. If teleradiotherapy (external beam radiotherapy - EBRT) is used, ionising radiation is generated outside the patient's body, usually with the use of linear accelerators. Brachytherapy is the second basic radiotherapy method, where the source of ionising radiation is introduced into the tumour or placed in its direct neighbourhood. The results of numerous clinical studies indicate that radiotherapy offers a possibility to cure patients in various clinical stages of the disease with a continuously decreasing rate of serious radiotherapy sequelae.

Key words: prostate cancer, radiotherapy, brachytherapy.

\section{Prostate cancer radiotherapy}

\author{
Anna Kulik, Mateusz Dąbkowski
}

Department of Brachytherapy, Centre of Oncology - Maria Skłodowska-Curie Memorial Institute in Warsaw

\section{Introduction}

Prostate cancer is one of the most commonly diagnosed malignancies in males, both in Poland and in other industrialised countries. According to the Polish National Cancer Register (Krajowy Rejestr Nowotworów) published in the year 2010, it accounted for $12.6 \%$ of newly diagnosed cancers and $7.5 \%$ deaths in Poland [1]. Cancer centres that treat this neoplasm use several treatment methods: surgery (radical prostatectomy, both open and laparoscopic), teleradiotherapy (external beam radiation therapy - EBRT) and brachytherapy (irradiation using a source of ionizing radiation placed into the tumour). Development of the EBRT technology, improvement of interstitial applications' quality as well as growing clinical experience enable optimisation of radiotherapy performed in all prostate cancer clinical stages. The purpose of this paper is to present general principles of qualification for treatment and treatment conduct in prostate cancer patients to doctors not specializing in medical or radiation oncology.

\section{Radiotherapy with use of external beams}

In the case of radiotherapy with external beams (teleradiotherapy; EBRT) the ionising radiation beam is generated outside the patient's body, usually in a machine called a linear accelerator. The principal mechanism of the effect of ionising radiation involves direct or indirect damage to the DNA chain of cancer cells. The cells whose genetic material was damaged lose their ability to divide and in consequence they undergo apoptotic death. Because of the presence of normal tissues within the irradiated volume, the dose of radiation necessary to cure a cancer cannot be administered as a single dose. In radiotherapy, differences in the division rate and ability to repair radiation damage between cancer cells and normal tissue cells are used. Therefore, the prescribed radiation dose is administered in the form of several to several tens of portions called fractions. Preparation of the treatment plan for a given patient involves definition of target volumes that should receive the prescribed dose and of critical organs, where the dose administered should be as low as possible. In the most popular method of prostate cancer radiotherapy - conformal three-dimensional EBRT - target volumes and critical organs are defined based on a computed tomography scan performed as the initial stage of treatment planning.

\section{External beam radiation therapy versus surgery}

In the recent literature, there are no reliable data enabling comparison of different prostate cancer treatment methods. The consensus of the National Institute of Health of 1988, concluding that prostatectomy and EBRT are equivalent treatment methods with respect to long-term survival [4], is still valid. However, these methods differ with respect to the toxicity profile. The decision to use one of these methods is taken based not only on the known prognostic factors for prostate cancer (baseline prostate-specific antigen [PSA] level, clinical stage, level or histopathological differentiation according to the Gleason scale), but also on the patient's preferences, his performance sta- 
tus, concomitant diseases and life expectancy. The results of two prospective randomised studies conducted in the 1970s and 1980s, comparing treatment efficacy between prostatectomy and EBRT, are available $[5,6]$. In the study of Akakura et al. with a 10-year follow-up, slightly better rates of overall survival, prostate cancer-specific survival and biochemical or clinical recurrence-free survival (67.9\% vs. $60.9 \%, 85.7 \%$ vs. $77.1 \%, 76.2 \%$ vs. $71.1 \%$, $83.5 \%$ vs. $66.1 \%$, respectively) were achieved in the prostatectomy group, as compared to the EBRT group. These differences were not statistically significant. However a statistically significant difference was found in favour of radiotherapy with respect to incontinence requiring more than 1 pad per day. In a study of Paulson et al., radical prostatectomy was associated with a statistically significant decrease of the biochemical recurrence rate, clinical recurrence rate and distant metastasis rate, as compared to EBRT. However, unequivocal conclusions for clinical practice cannot be drawn due to small sample sizes in analysed patient groups enrolled in particular studies, hormonal treatment of some patients, obsolete irradiation techniques and prescribing of total doses of ionising radiation that are currently considered inadequate. Recent non-randomised single- or multi-centre studies suggest similar efficacy of prostatectomy and radiotherapy in the treatment of prostate cancer. 2991 patients treated for prostate cancer clinical stage T1-T2 between 1990 and 1998 were enrolled in the most frequently quoted retrospective study conducted by researchers from the M.D. Andersen Cancer Center in Orlando. In this study, similar efficacy of radical prostatectomy, low dose rate (LDR) brachytherapy, EBRT with total doses of $>72$ Gy and combined treatment (EBRT plus LDR brachytherapy) was shown. The proportion of patients who survived 5 years without biochemical recurrence was $81 \%, 83 \%, 81 \%$, and $77 \%$, respectively. Only in the group of patients irradiated to a total dose lower than 72 Gy were poorer treatment results found. Only $51 \%$ of patients survived 5 years without biochemical recurrence [7]. An important aspect when comparing radical treatment methods - radiotherapy and prostatectomy - is treatment-related potency disturbances. A meta-analysis by Robinson et al. (2002) points to a higher probability of erection preservation in patients treated with radiotherapy, as compared to the surgery group. This probability in the period of 1 year is 0.76 for brachytherapy, 0.55 for EBRT, 0.34 for "nerve-sparing" prostatectomy, and 0.25 for standard radical prostatectomy [8].

\section{External beam radiation therapy techniques}

Up to the 1990s, conventional radiotherapy was the technique most frequently used in the radiation treatment of prostate cancer patients. In this technique, prostate location was based on fluoroscopy images. Some defined anatomical reference points were used for correct beam targeting: pubic symphysis, femoral heads and rectum and bladder filled with a contrast agent. Additionally, standard size treatment fields were used - large enough to minimise the risk of geographical miss resulting from limitations of imaging techniques. This made it necessary to include large volumes of critical organs adjacent to the prostate (rectum, bladder), as well as of the small intestine, in the irradiated area. With this technique, a dose of 65-70 Gy to the prostate was achievable, which is nowadays considered inadequate. Additionally, it was related to a higher rate of radiation-induced complications within critical organs. Despite its significant drawbacks, the conventional technique enabled satisfactory treatment results to be achieved. The 10-year prostate cancer-specific survival reached the level of $90 \%$ for low-grade adenocarcinoma, $75 \%$ for intermediate grade adenocarcinoma and 50\% for high-grade adenocarcinoma. The 5-year biochemical recurrence-free survival, evaluated in more recent studies when PSA level assessment was introduced into clinical practice, was $85 \%$ in patients with baseline PSA $<4 \mathrm{ng} / \mathrm{ml}$, $55 \%$ in patients with baseline PSA $4-10 \mathrm{ng} / \mathrm{ml}, 45 \%$ in patients with baseline PSA $10-20 \mathrm{ng} / \mathrm{ml}$ and $15 \%$ in patients with baseline PSA > $20 \mathrm{ng} / \mathrm{ml}$ [9].

Introduction of computed tomography into the treatment planning, development of computer planning systems and advanced technical solutions in linear accelerators completely changed the face of contemporary teleradiotherapy. At present, three-dimensional conformal radiotherapy is the standard technique in the treatment of patients with prostate cancer. This technique involves a CT scan performed in the patient to define the area to be treated. Based on this examination the radiation oncologist defines the so-called target volumes that should receive the prescribed dose, and critical organs where the dose should be as low as possible. Most commonly, the clinical target volume (CTV) includes the prostate or the prostate with a part of seminal vesicles. A relevant margin is added to the above volume (usually 5 to $15 \mathrm{~mm}$ ) that accounts for prostate mobility during irradiation and patient positioning inaccuracies during subsequent treatment fractions. The entire resulting planning target volume (PTV) should be covered by the prescribed isodose. The rectum, the bladder, the small intestine and the femoral heads are the most important critical organs with respect to radiotherapy of prostate cancer. The final radiotherapy treatment plan is prepared in a computer planning system based on definition of the CTV and PTV volumes, as well as of critical organs, in subsequent CT slices. Three-dimensional conformal radiotherapy enables, to a large extent, adjustment of the radiation dose distribution to the shape of the target volume with consideration of irradiated critical organs in the closest proximity. Owing to this technique, the volume of irradiated critical organs is limited by about $40-50 \%$ in comparison to the conventional technique [9]. A sample isodose distribution in a prepared radiotherapy plan and presentation of the patient's therapeutic position during irradiation with a linear accelerator are presented in Figure 1.

Intensity-modulated radiation therapy (IMRT) and other dynamic techniques are associated with change of parameters of the radiation beam during its emission. In the IMRT technique, during a single radiation fraction there is a continuous change of the shape of the irradiated field. This enables one to obtain practically any spatial dose distribution within the irradiated volume and thus better protection of critical organs. In comparison to three-dimensional conformal radiotherapy, IMRT also allows for administration of a higher radiation dose to the prostate with maintenance of tolerance doses for critical organs. 
Despite the fact that more and more refined EBRT techniques are used that enable one to obtain any dose distribution in the irradiated area, there is still the problem of organ motion between subsequent treatment fractions and of inaccuracies in precisely reproducing patient position on the treatment couch. Image-guided radiation therapy (IGRT) is an advanced treatment technique that helps to minimise this problem. It involves various imaging studies in the patient lying on the therapeutic couch directly before each radiation fraction and it is gradually becoming a standard in the treatment of prostate cancer patients. IGRT techniques include ultrasound-guidance systems, organ location with X-ray imaging of markers implanted into the prostate, radiolocation of special transponders implanted into the prostate, systems using location computed tomography, and use of markers placed on the patient's skin and detected with infrared cameras [10]. Image-guided radiation therapy enables further protection of critical organs and further dose escalation in the treated organ. This method is frequently used with other radiotherapy techniques (IMRT, dynamic technologies).

During the last 10 years, use of fractionated stereotactic radiotherapy in the treatment of prostate cancer patients has attracted immense interest in radiotherapy circles. Stereotactic radiotherapy involves administration of several high fraction doses to the prostate region; thus the treatment time is shortened to several days. To avoid complications within critical organs, stereotactic radiotherapy is associated with the necessity of very precise patient immobilisation, use of image-guided radiotherapy techniques (usually gold markers implanted into the prostate) and a complicated algorithm that enable "following" of the tumour location by the radiation beam. Preliminary data analysing acute and late toxicity and biochemical recurrence-free survival in patients treated with this method are very encouraging [11, 12].

\section{Contraindications to prostate cancer radiotherapy}

Contraindications to prostate cancer radiotherapy include history of inflammatory diseases of the large bowel (e.g. Crohn's disease, ulcerative colitis) and history of pelvic irradiation. Advanced patient's age and concomitant diseases affecting the patient's general condition may constitute relative contraindications and decisions about radiotherapy are then taken on a case-by-case basis.

\section{Interstitial brachytherapy using high dose rate sources}

Brachytherapy is the second basic radiotherapy method, where the source of ionising radiation is placed in the tumour (or in the tumour bed) or in its direct neighbourhood. Various radioisotopes (e.g. Ir-192, J-125, Co-60, Cs-137, Ru-106 and others), prepared specially for medical needs, are currently in clinical use. One of the important advantages of brachytherapy is the conformal nature of this treatment which allows for irradiation of the treated organ to a high total dose with significant limitation of the dose absorbed by adjacent critical organs. Brachytherapy methods' classification is based on various criteria: the way of application (interstitial, intracavitary, intraductal, superficial), time of source dwelling in the treated area (temporary, permanent) or dose rate of
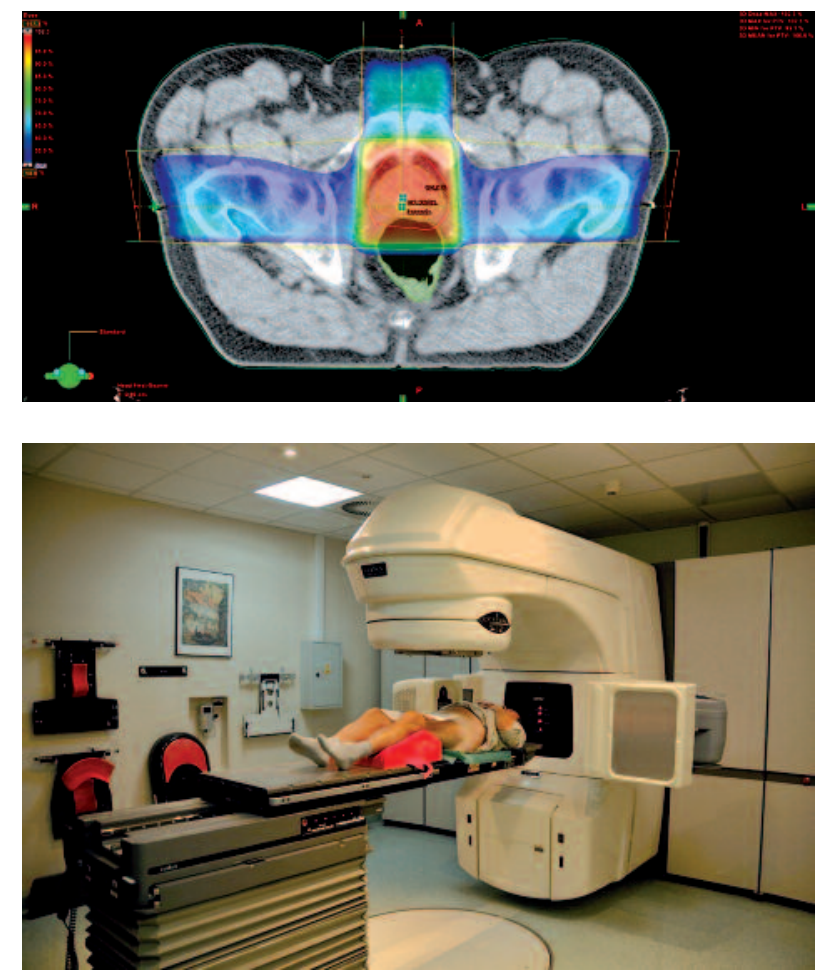

Fig. 1. A patient in the therapeutic position during irradiation with a high-energy linear accelerator, with a sample isodose distribution in a three-field technique

the source used (LDR - sources of low dose rate - 0.5-2 Gy/h; MDR - sources of medium dose rate - 2-12 Gy/h and HDR - sources of high dose rate - above $12 \mathrm{~Gy} / \mathrm{h}$ ). Advances in imaging and image reconstruction and thus in precise definition of target volumes (US, CT, MRI), introduction of the "stepping source" technique and afterloading into clinical practice as well as use of computer treatment planning and dose distribution optimization systems have all contributed to modernisation and popularisation of this field of oncology. Interstitial HDR brachytherapy is used in the treatment of prostate cancer both alone and in combination with EBRT [13]. Criteria of patient qualification for HDR brachytherapy alone or for combined radiotherapy differ in various protocols adopted by cancer centres. The recommendations of the American Brachytherapy Society (ABS) limit the use of HDR brachytherapy alone to low stage cases with favourable prognostic factors (clinical stage T1-2a, PSA level up to 10 mg/ml, Gleason score not higher than 6) [14]. The technique of the interstitial brachytherapy procedure is based on ultrasound imaging (transrectal ultrasound, TRUS) of the prostate. The application is usually conducted under subarachnoid anaesthesia, in the operating theatre setting. Imaging of the organ is integrated with a real time computer treatment planning system and allows for precise planning of both the number and location of interstitial guiding needles introduced transperineally, that constitute channels for source pass or, as in the LDR technique, a channel for introduction of permanent implants [13-16]. Particular stages of guiding needle implantation and irradiation and presentation of a final treatment plan are shown in Figures 2, 3 and 4. 


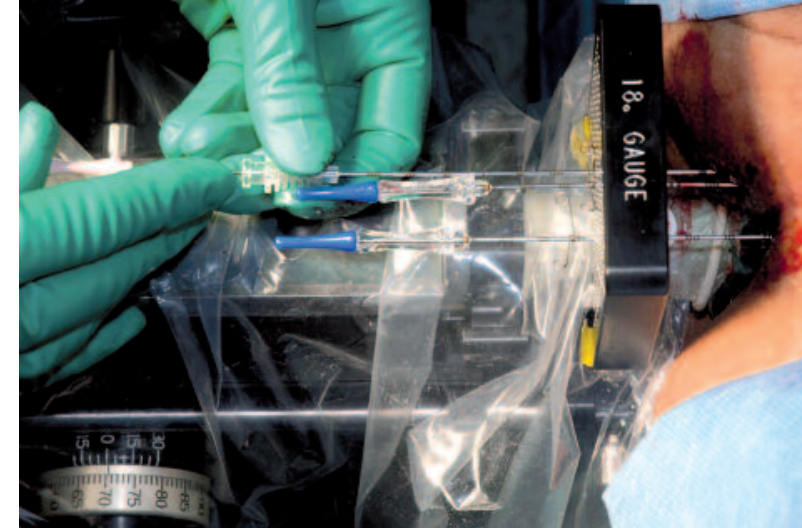

Fig. 2. TRUS-guided implantation of guiding needles containing seeds of radioactive $\mathrm{J}-125$

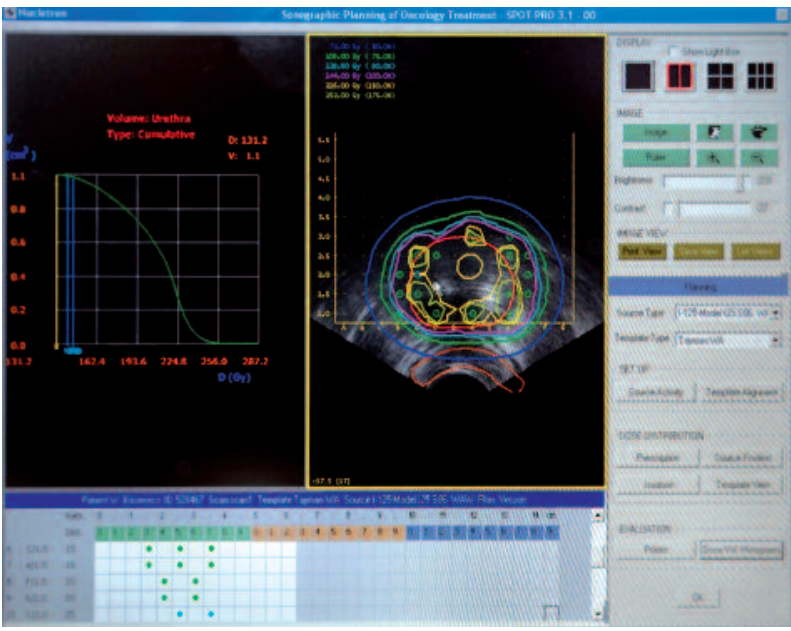

Fig. 4. Radiotherapy plan prepared in a computer treatment planning system - the LDR technique

\section{Low dose rate interstitial brachytherapy - permanent implants}

Seeds containing J-125 are currently the most popular radioactive sources used for permanent applications. It is a $\beta$ and $\gamma$ radiation emitter with a clinically and biologically favourable half-life. The following patients are qualified for interstitial treatment with low dose rate sources: early stage prostate cancer without unfavourable risk factors of local recurrence of the disease and distant metastasis - clinical stage T1a, T1b, T1c, T2a NOMO, Gleason score up to 6, PSA level $10 \mathrm{ng} / \mathrm{ml}$. Prostate volume determined in TRUS examination should not exceed $50 \mathrm{ml}$. Contraindications to prostate irradiation with the above method, besides clinical factors, is location of part of the organ behind the pubic symphysis, which prevents appropriate application of guiding needles with the sources; status post the TURP procedure with a wedge resection of periurethral parts of the prostate; life expectancy less than 5 years; patient refusal to undergo the suggested treatment; other serious concomitant disease precluding anaesthesia or appropriate patient positioning in the therapeutic position, with abduction and flexion of the lower limbs at the hip joints; and a history of pelvic radiother-

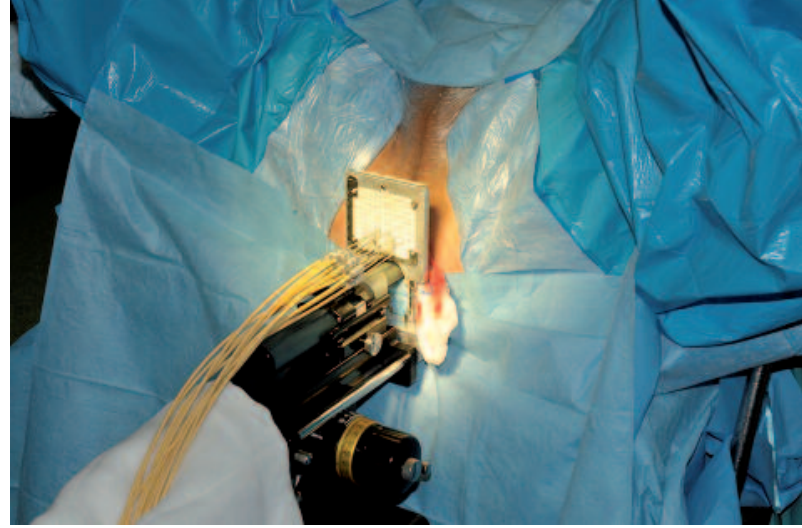

Fig. 3. HDR technique. Interstitial guiding needles connected with connecting channels with the HDR machine

apy [9]. Technically, the procedure of implantation of seeds with radioactive sources is similar to the HDR technique. The most common procedure-related complications are haematuria and acute urinary retention resulting from prostate swelling and requiring urinary catheter insertion. In the analysed groups of patients, the rate of these complications does not exceed 10\% [17]. Acute and late radiation-induced reactions are presented in Table 1.

\section{Combined radical radiotherapy}

Clinical observations suggest that local failure is the main cause of radiotherapy failure in the treatment of prostate cancer [18-20]. This resulted in introduction of treatment protocols escalating the total dose of ionising radiation into clinical practice. One method of local dose escalation is combination of EBRT and interstitial brachytherapy. The results of studies on total dose escalation by combining EBRT and HDR brachytherapy have shown that such combination has an effect on increase of the local cure rate, prolongation of the time to biochemical recurrence and overall survival [21-23]. IRPCa patients (with intermediate risk of local recurrence) and HRPCa patients (with high risk of local recurrence) (>T2a; PSA > $10 \mathrm{ng} / \mathrm{ml}$; histopathological grade above 6 according to the Gleason scoring system) have particular benefit from this treatment method [24, 25].

In 2005, the European Brachytherapy Group (GEC/ESTRO) and the European Urology Society (EAU) developed common criteria of patients' qualification for combined radiotherapy. According to these criteria, the candidates for combined radiotherapy are patients with local stage T1b-T3b, with any Gleason score, with baseline PSA above $50 \mathrm{ng} / \mathrm{ml}$, and with no distant metastases. As contraindications, the following are listed: life expectancy lower than 5 years, involvement of the external sphincter of the bladder neck, and presence of cancer metastases in regional lymph nodes or of distant metastases. Additionally, as relative contraindications, the following are considered: prostate volume above $60 \mathrm{ml}$, history of partial transurethral prostate resection within 6 months before the treatment, the distance between the rectal wall and prostate capsule below $5 \mathrm{~mm}$ in TRUS, serious symptoms of infravesical obstruction, technically impossible implantation (pubic symphysis) or problems with patient positioning in 
a "gynaecological" position (e.g. coxarthrosis), and anaesthesiological contraindications.

\section{Radiation-induced complications}

Radiation-induced complications resulting from reaction of tissues to the ionising radiation affect both the prostate itself and the neighbouring organs, first of all the rectum and the bladder. The most common clinical symptoms are the following: frequent urination, urgency, urinary incontinence, haematuria and pain, both urination-related and spontaneous. Their severity is related, among other things, to the total dose administered, use of increased fraction dos- es in the treatment regimen and large volume of the irradiated tissue block. Within the post-treatment follow-up both the so-called acute (during radiotherapy and up to three months after its completion) and late (above 90 days from radiotherapy completion) radiation-induced reactions are assessed. Their qualitative and quantitative severity is graded from 0 to 4 . The symptoms of acute and late radiationinduced reactions within the urinary system by severity grade are presented in Table 1.

Current recommendations concerning the role of radiation therapy in treatment of subsequent risk groups of prostate cancer patients are summarized in Table 2.

Table 1. Grading of acute and late urinary system radiation-induced reactions

\begin{tabular}{|c|c|c|}
\hline & $\begin{array}{l}\text { Acute radiation-induced reactions } \\
\text { according to the EORTC scale }\end{array}$ & $\begin{array}{l}\text { Late radiation-induced reactions according to the } \\
\text { RTOG/SOMA scale }\end{array}$ \\
\hline I. & $\begin{array}{l}\text { Micturition and nycturia are twice as frequent } \\
\text { as before the treatment. } \\
\text { The patient reports painful or difficult urination } \\
\text { and/or urgency. }\end{array}$ & $\begin{array}{l}\text { Episodes of urinary incontinence occur less frequently than once a week. } \\
\text { Nocturnal micturition is reported by the patient up to two times per night. } \\
\text { Microscopic haematuria is found. } \\
\text { Minor mucosal atrophy and single telangiectasias are found on cystoscopy. }\end{array}$ \\
\hline II. & $\begin{array}{l}\text { Micturition and nocturnal micturition at } \\
\text { one-hour intervals. } \\
\text { Painful or difficult urination and/or urgency } \\
\text { severity requires NSAID prescription. }\end{array}$ & $\begin{array}{l}\text { Episodes of urinary incontinence occur less frequently than once a day. } \\
\text { The patient reports nycturia of a higher frequency than twice per night. } \\
\text { Generalized mucosal telangiectasias are found in the bladder on cystoscopy. } \\
\text { Gross haematuria appears periodically. The patient requires non-steroidal } \\
\text { analgesics. }\end{array}$ \\
\hline III. & $\begin{array}{l}\text { Micturition and nocturnal micturition more frequent } \\
\text { than every hour. } \\
\text { Painful or difficult urination and urgency severity } \\
\text { requiring prescription of narcotic analgesics. } \\
\text { Gross haematuria is found. }\end{array}$ & $\begin{array}{l}\text { The patient reports permanent urinary incontinence. } \\
\text { Hourly nycturia, procedures of urinary duct widening are necessary. } \\
\text { Bladder capacity decrease below } 150 \mathrm{ml} \text { is recorded in urodynamic tests. } \\
\text { Haematuria severity needs blood transfusions. Genitourinary fistulas are } \\
\text { found on clinical examination. } \\
\text { The patient requires narcotic analgesics. }\end{array}$ \\
\hline IV. & $\begin{array}{l}\text { Symptoms as above. } \\
\text { Haematuria requiring blood transfusion. } \\
\text { Ulceration or necrosis of the bladder or rectal wall } \\
\text { is diagnosed. }\end{array}$ & $\begin{array}{l}\text { Due to major stenosis of urinary ducts there is a need of permanent } \\
\text { catheterisation or cystectomy. } \\
\text { Severe haemorrhagic inflammation or ulceration of the bladder mucosa } \\
\text { requires permanent catheterization or cystectomy. }\end{array}$ \\
\hline
\end{tabular}

Table 2. Radical radiotherapy in the low, intermediate and high local recurrence risk groups according to the guidelines of the National Comprehensive Cancer Network (NCCN)

\begin{tabular}{|c|c|c|}
\hline Group & Group characteristics according to the NCCN & Suggested treatment regimen including radiotherapy \\
\hline $\begin{array}{l}\text { Low risk of local } \\
\text { recurrence }\end{array}$ & $\begin{array}{l}\text { Clinical stage T1-T2a and Gleason score } \\
\leq 6 \text { and PSA level }<10 \mathrm{ng} / \mathrm{ml}\end{array}$ & $\begin{array}{l}\text { Conformal or IMRT 3D radiotherapy, using IGRT techniques, with } \\
\text { total dose escalation to a level of > } 74 \text { Gy (e.g. brachytherapy), } \\
\text { without pelvic nodal irradiation, without hormone therapy, } \\
\text { or } \\
\text { LDR brachytherapy (seeds), optionally with a 3-month } \\
\text { neoadjuvant hormonal blockade }\end{array}$ \\
\hline $\begin{array}{l}\text { Intermediate risk } \\
\text { of local recurrence }\end{array}$ & $\begin{array}{l}\text { Clinical stage T2b-T2c or Gleason score } 7 \\
\text { or PSA level } 10-20 \mathrm{ng} / \mathrm{ml}\end{array}$ & $\begin{array}{l}\text { Conformal or IMRT 3D radiotherapy, using IGRT techniques, } \\
\text { with dose escalation up to } 76-80 \text { Gy (e.g. brachytherapy), } \\
\text { with 4-6-month hormonal blockade; pelvic nodal irradiation may } \\
\text { be considered }\end{array}$ \\
\hline $\begin{array}{l}\text { High risk of local } \\
\text { recurrence }\end{array}$ & $\begin{array}{l}\text { Clinical stage T3a or Gleason score 8-10 } \\
\text { or PSA level > } 20 \mathrm{ng} / \mathrm{ml}\end{array}$ & $\begin{array}{l}\text { Conformal or IMRT 3D radiotherapy, using IGRT techniques, } \\
\text { with dose escalation up to } 76-80 \text { Gy (e.g. brachytherapy), } \\
\text { with 2-3-year hormonal blockade, with pelvic nodal irradiation }\end{array}$ \\
\hline
\end{tabular}




\section{References}

1. Wojciechowska U, Didkowska J, Zatoński W. Nowotwory złośliwe w Polsce w 2008 roku. Wydawnictwo Centrum Onkologii - Instytut im. M. Skłodowskiej-Curie; Warszawa 2010.

2. Awan SB, Hussain M, Dini SA. Historical review of interstitial prostate brachytherapy Iran J Radiat Res 2008; 5: 153-68.

3. Ray GR, Cassady JR, Bagshow MA. Definitive radiation therapy of carcinoma of the prostate. A report on 15 years of experience. Radiology 1973; 106: 407-18.

4. Consensus statement: the Management of Clinically Localized Prostate Cancer. National Institutes of Health Consensus Development Panel [no authors listed]. NCI Monogr 1988; 7: 3-6.

5. Akakura K, Suzuki H, Ichikawa T, et al. A randomized trial comparing radical prostatectomy plus endocrine therapy versus external beam radiotherapy plus endocrine therapy for locally advanced prostate cancer: results at median follow-up of 102 months. Jpn J Clin Oncol 2006; 36: 789-93.

6. Paulson DF, Lin GH, Hinshaw W, Stephani S. Radical surgery versus radiotherapy for adenocarcinoma of the prostate. J Urol 1982; 128: 502-4.

7. Kupelian PA, Potters L, Khuntia D, et al. Radical prostatectomy, external beam radiotherapy $<72$ Gy, external beam radiotherapy $>$ or = 72 Gy, permanent seed implantation, or combined seeds/external beam radiotherapy for stage T1-T2 prostate cancer. Int J Radiat Oncol Biol Phys 2004; 58: 25-33.

8. Robinson JW, Moritz S, Fung T. Meta-analysis of rates of erectile function after treatment of localized prostate carcinoma. Int J Radiat Oncol Biol Phys 2002; 54: 1063-8.

9. Nilsson S, Norlén BJ, Widmark A. A systematic overview of radiation therapy effects in prostate cancer. Acta Oncol 2004; 43: 316-81.

10. Miszczyk L, Leszczyński W, Szczepanik K, et al. Comparison of two image guided radiation therapy (IGRT) methods used for prostate cancer patients-CBCT and 2D-2D kV. Przegl Lek 2008; 65: 315-20.

11. King CR, Brooks JD, Gill H, Presti JC Jr. Long-term outcomes from a prospective trial of stereotactic body radiotherapy for low-risk prostate cancer. Int J Radiat Oncol Biol Phys 2011 Feb 5.

12. Aznar MC, Petersen PM, Logadottir A, et al. Rational radiotherapy for prostate cancer in clinical practice. Radiother Oncol 2010; 97 : 480-4.

13. Kanikowski M, Skowronek J, Milecki P, et al. Brachyterapia HDR raka gruczołu krokowego. Urol Pol 2007; 60: 5-11.

14. Kovacs G, Potter R, Loch T, et al. GEC/ESTRO-EAU recommendations on temporary brachytherapy using stepping sources for localized prostate cancer. Radiother Oncol 2005; 74: 137-48.

15. Astrom I, Pedersen D, Mercke C i wsp. Long-term outcome of high dose rate brachytherapy of localized prostate cancer. Radiother Oncol 2005; 74: 157-61.

16. Hoskin P. High dose rate brachytherapy for prostate cancer. Cancer/Radiotherapie 2008; 12: 512-4.

17. Akimoto T, Katoh H, Noda S. Acute genitourinary toxicity after high dose rate (HDR) brachytherapy combined with hypofractionated external-beam radiation therapy for localized prostate cancer: second analysis to determine the correlation between the urethral dose in HDR brachytherapy and the severity of acute genitourinary toxicity. Int J Radiat Oncol Biol Phys 2005; 63: 472-8.

18. Bachand F, Martin AG, Beaulieu I, et al. An eight-year experience of HDR brachytherapy boost for localized prostate cancer: biopsy and PSA outcome. Int J Radiat Oncol Biol Phys 2009; 73: 679-84.

19. Carpenter TJ, Forsythe K, Kao J, et al. Outcomes for patients with extraprostatic prostate cancer treated with trimodality therapy, including brachytherapy, external beam radiotherapy, and hormone therapy. Brachytherapy 2011; 10: 261-8.

20. Chen Y-C, Chuang C-K, Hsieh M-L. High-dose-rate brachytherapy plus external beam radiotherapy for $\mathrm{T} 1$ to $\mathrm{T} 3$ prostate cancer: an experience in Taiwan. Urology 200; 70: 101-5.

21. Demanes DJ, Rodriguez RR, Schour L i wsp. High-Dose-Rate intensity-modulated brachytherapy with external beam radiotherapy for prostate cancer: California Endocurietherapy's 10-year results. Int J Radiat Oncol Biol Phys 2005; 61: 1306-16.

22. Deutsch I, Zalefsky MJ, Zhang Z, et al. Comparison of PSA relapsefree survival In patients treated with ultra-dose IMRT versus com- bination HDR brachytherapy and IMRT. Brachytherapy 2010; 9: 313-8.

23. Galalae RM, Martinez AA, Mate T, et al. Long-term outcome by risk factors using conformal high dose-rate brachytherapy (HDR-BT) boost with or without neoadjuvant androgen suppression for localized prostate cancer. Int J Radiat Oncol Biol Phys 2004; 58: 1048-55.

24. Ghadjar P, Rentsch CA, Isaak B, et al. Urethral toxicity vs. Cancer control- Lessons to be learned from high-dose rate brachytherapy combined with intensity-modulated radiation therapy in intermedia and high-risk prostate cancer. Brachytherapy 2011; 10: 286-94.

25. Hoskin P. High dose rate brachytherapy boost treatment in radical radiotherapy for prostate cancer. Radiother Oncol 2000; 57: 285-8.

\section{Address for correspondence}

Anna Kulik MD, PhD

Department of Brachytherapy

Centre of Oncology - Maria Skłodowska-Curie Memorial

Institute in Warsaw

Roentgena 5

02-781 Warsaw

e-mail: anak7@wp.pl 